\title{
A novel methodology for time-domain characterization of a full anechoic chamber for antennas measurements and exposure evaluation
}

\author{
Chakib Taybi, Mohammed Anisse Moutaouekkil, Bachir Elmagroud, Abdelhak Ziyyat \\ Electronic and Systems Laboratory, Faculty of Sciences, Mohammed First University, Oujda, Morocco
}

\begin{tabular}{l}
\hline Article Info \\
\hline Article history: \\
Received Aug 11, 2020 \\
Revised Dec 15, 2020 \\
Accepted Jan 19, 2021 \\
\hline
\end{tabular}

Keywords:

Anechoic chamber Antennas measurement Autoregressive filter Electromagnetics measurement Exposure evaluation Finite technique method Time domain simulation

\begin{abstract}
In this paper we present a novel methodology for time-domain characterization of a full anechoic chamber using the finite integral method. This approach is considered fast, accurate and not intensive for computer resources. The validation of this approach is carried out on CST-microwave studio for a full anechoic chamber intended for antennas measurement applications and electromagnetic exposure evaluation for cellular network. Low, medium and high gain sources are used in this study. The simulations are realized on a personal computer of medium performances (i7 CPU and $16 \mathrm{~GB}$ of RAM). The stability and the convergence of our approach are obtained thanks to local mesh and auto-regressive linear filtering techniques. The minimization of the simulation time is based on use of the Huygens sources in the place of the antennas. The maximum error of the chamber as well as the wave depolarization into the chamber are at one with the previous work and the catalogs of the principles chambers manufacturers for the proposed tests in this paper. The Full simulations time is about 15 hours in average.
\end{abstract}

This is an open access article under the CC BY-SA license.

\section{Corresponding Author:}

Chakib Taybi

Electronic and Systems Laboratory

Faculty of Sciences

Mohammed First University

P.O. Box: 717, Oujda, Morocco

Email: ch.taybi@ieee.org

\section{INTRODUCTION}

Today, anechoic chamber becomes a perfect solution for antenna measurements [1] and for the assessment of exposition to electromagnetic fields (EMF) [2]. They allow to reproduce OATS conditions [3] in a limited, closed and protected environment. Different forms and types of anechoic chamber are presented in previous work $[4,5]$. However, the rectangular form is the most one used for antennas measurement and EMF evaluation.

Typically, an anechoic chamber essentially consists of a shielding structure [6], protects the volume test, called quiet zone generally, from the noise sources and a matrix of electromagnetic absorber [7] minimizes the reflections on the walls of the chamber. Special antennas type, called probes [8], are used to sample the EM field radiated by the antenna under test.

The characterization of the anechoic chambers and its constituent dates back to the 1975 [4]. In this era, the Ray-Tracing $[9,10]$ method was used to model objects whose dimensions are higher than the 
wavelength. Years later, other methods benefit, from the computing and storage power of personal computer, and from the development of numerical codes, which are implemented for the characterization of chambers whose dimensions are in the lower of wavelength. In this context, the finite elements method (FEM) presented in $[11,12]$ was used for modeling a full anechoic chamber for antenna measurements. The simulations were launched on a super calculation station (dual Xeon CPU and 256 GB of RAM) and they required 196 GB of memory and a calculation time of 15 hours for a frequency of $1 \mathrm{GHz}$. For higher frequencies the proposed method requires more memory and more of the computing time.

The finite differences in time domain FDTD were also used to analyze the behavior of a full anechoic and compact chambers $[13,14]$. The obtained results show that this method is more suitable for dielectric objects such as electromagnetic absorbers. For metallic structures such as walls, probes, antennas and scanners system, it requires a very fine mesh. Both a larger memory space and a longer computation time are needed for this purpose. An alternative solution based on the transmission lines matrix (TLM) was presented by Gwenael [15] in the order to characterize a full and a semi-anechoic chamber. The obtained results show the need for a special type of perfect matched layers (PML) [15] to reduce the IT resource requirements and also the simulation time. Therefore, the simulations were lunched out on a personal computer and lasted about 150 hours in average. In addition, the work presented in $[16,17]$ demonstrates the need to combining TLM with an other method to ensure accuracy at lower frequencies.

Also, vision-based technology are used for modeling many physical systems [18]. These methods proved their utility for wireless channel characterization [19], vehicle problem and RFID signal measurements [20]. However, for electromagnetic problems, these methods reverent incapable to describe Maxwell equations in a finite cell and the boundary conditions for a large structures such as anechoic chamber.

In order to optimize IT resource requirements and to properly control the simulation time, we have presented in [21] a novel approach for the characterization of a full anechoic chamber. Based on the finite integrals method (FIT) and on linear regression filtering technique. This novel method results show that it's accurate, fast and not intensive in computing resources. The validation was performed for a chamber for antenna measurements and with a medium gain source. In this paper, we are expanding the validation domain of our novel approach. The chamber behavior was evaluated for a low, medium and high gain sources. This three sources types, represent the possible cases encountered in an anechoic chamber for antenna measurements. The chamber error and the wave depolarization in this later was evaluated as function of the three proposed sources. The calculation time and the memory size used are also compared in this paper.

\section{METHOD AND MATERIAL}

\subsection{Method}

The characterization of the structure of the full anechoic chamber and its elements was carried out with the finite integrals technique (FIT) $[22,23]$ available under the CST microwave studio suite. This technique allows Maxwell equations resolution in the integral form described by (1-4).

$$
\begin{gathered}
\oint_{\partial A} E \cdot d S=-\int_{A} \frac{\partial B}{\partial t} \cdot d A \\
\oint_{\partial A} H \cdot d S=-\int_{A}\left(J+\frac{\partial D}{\partial t}\right) \cdot d A \\
\oint_{\partial V} B \cdot d A=0 \\
\oint_{\partial V} D \cdot d A=\int_{V} \rho \cdot d V
\end{gathered}
$$

A cubic elementary mesh as shown in Figure 1 discretization is applied to the structure of the chamber or to one of its elements. Equation (1) can be written on a mesh in the form of (5).

$$
\hat{e}_{l}+\hat{e}_{k}+\hat{e}_{j}+\hat{e}_{i}=-\frac{d}{d t} \hat{b}_{n}
$$


The scalars $\hat{e}$ and $\hat{b}_{n}$ represent respectively the E field and the B flux on the edge and the face of a mesh cell. Equation (5) can also be written as (6). By similar demonstration, the (2), (3) and (4) can be written in the form of the (7), (8) and (9).

$$
\begin{gathered}
C . \hat{e}=-\frac{d}{d t} \cdot \hat{b}_{n} \\
\bar{C} . \hat{h}=\hat{j}+\frac{d}{d t} \cdot \hat{b} \\
S . \hat{b}=0 \\
\bar{S} . \hat{d}=\rho
\end{gathered}
$$

Where $\mathrm{C}$ and $\mathrm{S}$ are topological matrix $(-1,0,1)$ and represent the edge-to-face incidence.

Time domain resolution (or transit solver in CST) of the previous (6), (7), (8), (9) allows the field $e$ and the flux $b$ to be written in an iterative form Figure 2 given by (10), (11).

$$
\begin{gathered}
h^{i+1}=h^{i}-\Delta t \cdot M_{\mu}^{-1} \cdot C \cdot e^{i+\frac{1}{2}} \\
e^{i+\frac{3}{2}}=e^{i+\frac{1}{2}}+\Delta t \cdot M_{\epsilon}^{-1}\left[\bar{C} \cdot h^{i+1}-j^{i+1}\right]
\end{gathered}
$$

Where $M_{\epsilon}$ and $M_{\mu}$ represent the discrete matrix of permittivity and permeability.

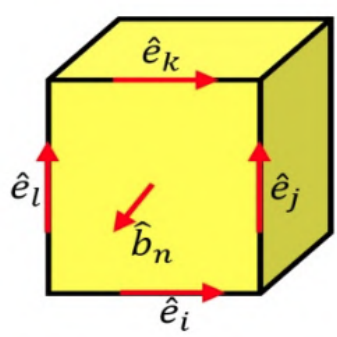

Figure 1. Cubic elementary mesh discretization

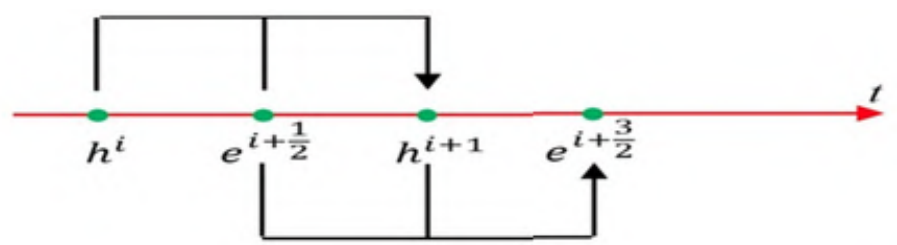

Figure 2. Iterative time domain resolution of Maxwell equation

For a large and highly resonant structure (case of an anechoic chamber), the FIT becomes unstable and more intensive both in computing resources and in computing time due to the very fine mesh requirements. Calculation time itself is a function of the number of computation cells, of the process of excitation of the probe and the AUT. To overcome this problem, reduce and minimize both the IT resources and also the computation time, the EM field of the source is calculated on another simulation file and with an adequate mesh. These EM fields are calculated separately and are exported into the main simulation file as a Huygens sources. This technique reduce significantly the simulation as will discussed in the results part.

However, the instability and divergence of FIT remains one of the main problems dealt with in this method. This is mainly due to the high resonance of the structure and to the FIT conditioning problems. 
To solve this problem, we have used auto-regressive (order 3) filter in parallel [24, 25]. This filter types are capable of calculating spectra (12) for time signals which have not yet reached their equilibrium state. These time signals are essentially those of the EM field monitors.

$$
x(n)=-\sum_{k=1}^{p} a_{k} x(n-k)
$$

Where $\mathrm{p}$ is the filter order.

\subsection{Simulation materials}

In order to validate our approach, we studied in this paper the behavior of a full rectangular anechoic chamber for near and/or far field antenna measurement applications. The dimensions of this chamber are $500 \times 300 \times 300(\mathrm{~L}(\mathrm{~cm})-\mathrm{W}(\mathrm{cm})-\mathrm{H}(\mathrm{cm}))$ and it consists as shows the Figure 3 of the following elements:

- Shielding structure: Modeled by a metal cage of conductivity $5.8 .10^{8} \mathrm{~S}_{\mathrm{m}}^{-1}$ and thickness of $0.5 \mathrm{~cm}$. This cage has five apertures associated to their waveguides for the passage of cables. The shielding efficiency (SE) of this cage was studied in detail in our previous article [21].

- Electromagnetic absorbers: Two types of absorbers were used in this study. The first one is flat absorbers (AT), used in the corners of the chamber. The second one is pyramidal shape (APM21, APM30 and APM 45), used in high incidence areas. The dimensions and the reflectivity of the absorbents used are reported in [21].

- Antennas sources: Three types of antennas are tested in this paper. Half-wave dipole, open ended rectangular wave guide (OERWG) and finally a pyramidal horn. These types of sources are the most used in an anechoic chamber for antennas measurement applications. The dimensions and the radiation pattern of the antenna sources at a frequency of $1 \mathrm{GHz}$ are reported in literature and our previous works [5, 21].

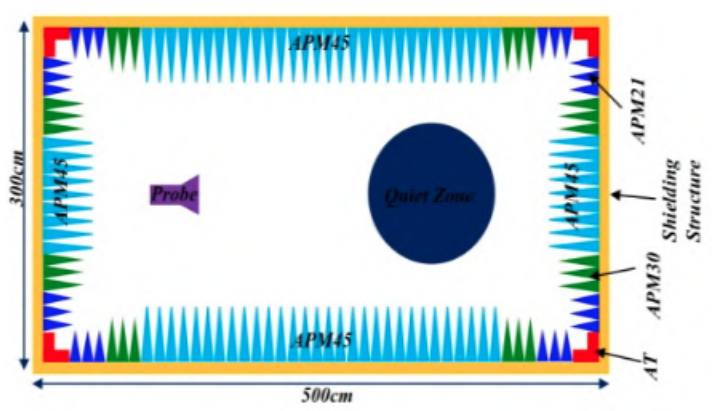

(a)

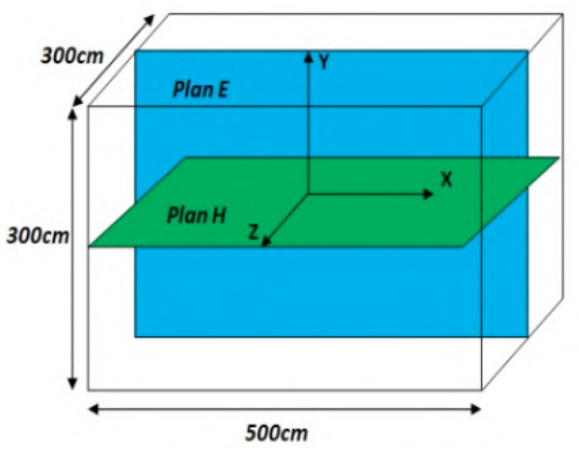

(b)

Figure 3. Illustration of the full chamber form and measurements planes,

(a) dimensions and the components of the chamber, (b) Measurement planes inside the chamber

Because our method is fast and doesn't require a lot of computer resources, the simulations are launched on a personal computer of $3.4 \mathrm{GHz}$ of CPU and $16 \mathrm{~GB}$ of RAM. A recent version of CST microwave studio is installed on this computer.

The performances of the anechoic chamber were evaluated using two principles parameter. The first one is the chamber error (13), defined by the ratio of the field radiated by the source inside the chamber and the field radiated in free space (without chamber). The second one is the axial ratio, represents the wave depolarization (14) inside the chamber. This last one is defined by the ratio between the vertical to the horizontal component of the field radiated inside the chamber. These two parameters (error and axial ratio) are generally given in $\mathrm{dB}$ value ( $0 \mathrm{~dB}$ is assigned to a perfect chamber). Practically, a value lower than $4 \mathrm{~dB}$ is generally supported by antennas measurement systems.

$$
\operatorname{Err}(d B)=\left|\sqrt{\frac{\sum \mid \text { Field }\left._{\text {chm }}\right|^{2}}{\sum \mid \text { Field }\left._{\text {free }}\right|^{2}}}\right|
$$




$$
A R(d B)=\left|\sqrt{\frac{\sum \mid \text { Field }\left._{H}\right|^{2}}{\sum \mid \text { Field }\left._{V}\right|^{2}}}\right|
$$

The components of the field inside the chamber and in the free space are calculated for a frequency of $1 \mathrm{GHz}$ and on two orthogonal planes (Plane E and Plane $\mathrm{H}$ ) localized at center of the chamber as shown the Figure 3.

\section{RESULTS AND DISCUSSION}

In this section, we discuss the results validating our novel approach. As mentioned in the previous section, the first step in our approach consists in calculating the Huygens sources necessary to characterize the EM field in the chamber. The Table 1 summarizes for each source the directivity $\mathrm{D}$ in $\mathrm{dBi}$, the number of cells mesh, the size of the memory and the simulation times.

Table 1. Resume of Huygens calculation for each source

\begin{tabular}{lcccr}
\hline Source & D & Mesh & RAM & Time \\
\hline Dipole & 2.5 & 2560 & $\leq 1 G B$ & $\leq 1 \mathrm{~min}$ \\
OERWG & 6.2 & 15450 & $1.3 \mathrm{~GB}$ & $5 \mathrm{~min}$ \\
Horn & 15 & 265400 & $3.2 \mathrm{~GB}$ & $15 \mathrm{~min}$ \\
\hline
\end{tabular}

To validate our approach and to analyze the behavior of the chamber, we present on the Figures 4-7, for each of the sources, the error (13) and the axial ratio (14), in the full length of the chamber and also in its quiet zone. The chamber error and the axial ratio are analyzed on both planes and also as function of the source polarization.

For each of the sources studied in this paper, the obtained results show that the error is maximum in the structure of the absorbers, in zones with great reflections and in areas near to the radiating source. This error, which varies as function of the source and the previous regions, is about of $50 \mathrm{~dB}$ for both horizontal and vertical polarizations. In the quiet zone of the chamber, the maximum error is $5 \mathrm{~dB}, 1 \mathrm{~dB}$ and $3 \mathrm{~dB}$ for dipole, OERWG and horn source respectively for the two polarizations.

In the same way, the axial ratio, which represents the depolarization of the wave in the chamber is maximum in the absorbers structures and near to the source and it is minimum in the quiet zone in the chamber. For the dipole source, it is by means of $3 \mathrm{~dB}, 1.2 \mathrm{~dB}$ for the OERWG and $2 \mathrm{~dB}$ for the horn, for horizontal as for vertical polarization.

Analysis of the results presented in the figures below shows that the dimensions of the chamber proposed in this study are compatible with the sources with moderate directivity. For other types of sources, a calibration process is necessary to correct the error and the depolarization of the wave in the chamber. On the other hand, the chamber error and the axial ratio calculated with our method are comparable with the results published in the bibliography $[11,12,26]$ and in the catalogs of the chamber manufacturers [27, 28]. Unlike other methods, which require either good hardware or enormous computation time, our simulations are performed on a desktop computer with acceptable performances. The Table 2 summarizes for each source the total number of cells, the memory required and finally the total calculation time.

Table 2. Resume simulations details of each sources

\begin{tabular}{lccr}
\hline Source & Mesh & RAM & Time \\
\hline Dipole & 58000000 & $12.5 \mathrm{~GB}$ & $12.5 \mathrm{~h}$ \\
OERWG & 61000000 & $14.8 \mathrm{~GB}$ & $14.4 \mathrm{~h}$ \\
Horn & 64000000 & $15.4 \mathrm{~GB}$ & $5.5 \mathrm{~h}$ \\
\hline
\end{tabular}

The results presented in the Table 2 show the capacity and the solidity of our new approach for the characterization of a full anechoic chamber on a machine with acceptable performances, in a reasonable time and with an error and an axial ratio tolerable by the most of the antenna measurement systems. The slight difference of the number of mesh cells, memory and of the computation time from source to another is mainly due to the spherical surface used to calculate the Huygens sources. 


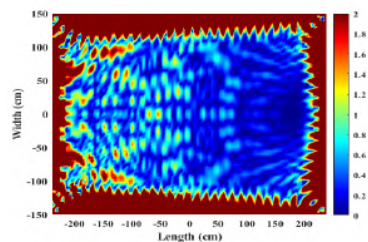

Err(PlanE,Chm,H)

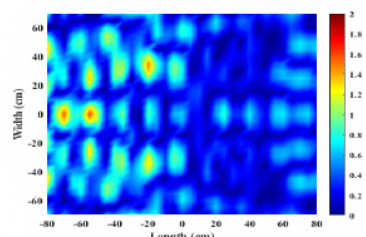

Err(PlanE,Zone,H)

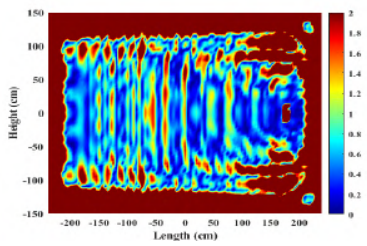

Err(PlanH,Chm,H)

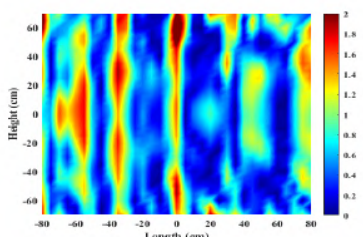

Err(PlanH,Zone,H)

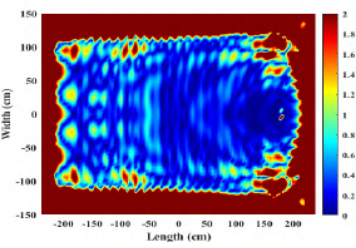

Err(PlanE,Chm,V)

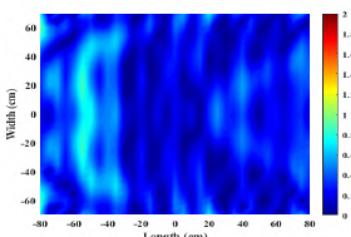

Err(PlanE,Zone,V)

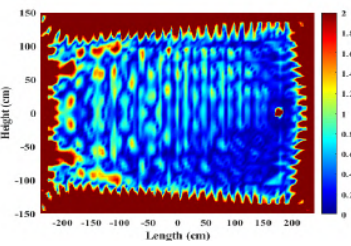

Err(PlanH,Chm,V)

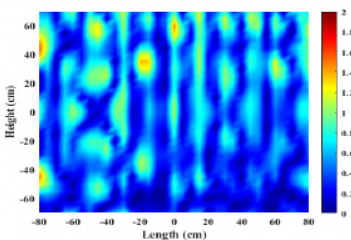

$\operatorname{Err}($ PlanH,Zone, $\mathrm{V})$

Figure 4. Error in the full length of chamber and in the quiet zone at $1 \mathrm{GHz}$ for a dipole source

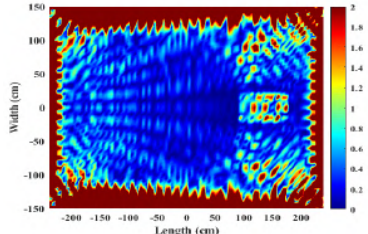

Err(PlanE,Chm,H)

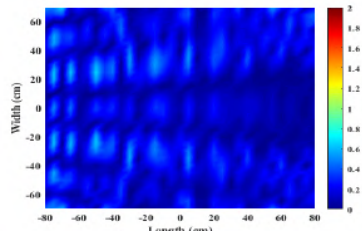

Err(PlanE,Zone,H)

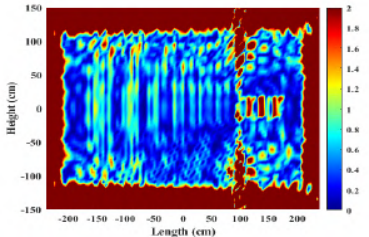

Err(PlanH,Chm,H)

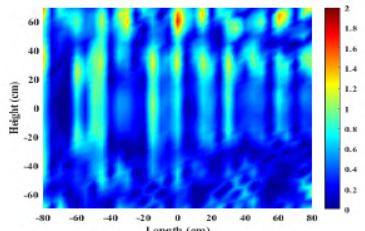

Err(PlanH,Zone,H)

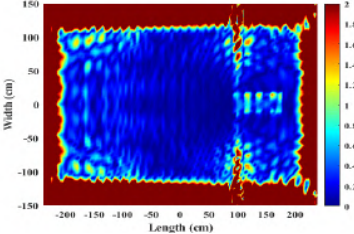

Err(PlanE,Chm,V)

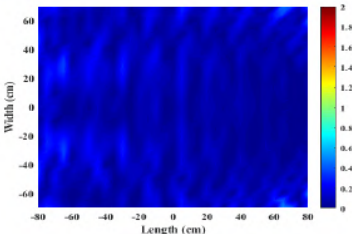

Err(PlanE,Zone, V)

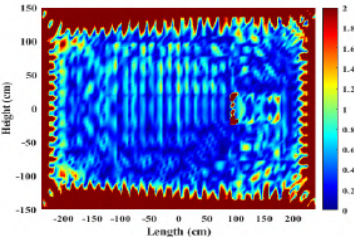

Err(PlanH,Chm,V)

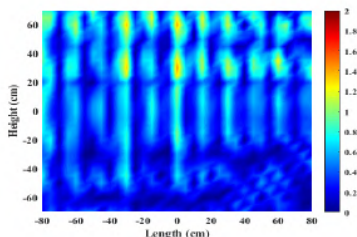

Err(PlanH,Zone,V)

Figure 5. Error in the full length of chamber and in the quiet zone at $1 \mathrm{GHz}$ for a OERWG source

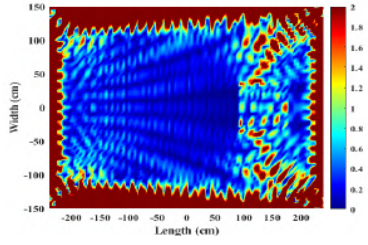

Err(PlanE,Chm,H)

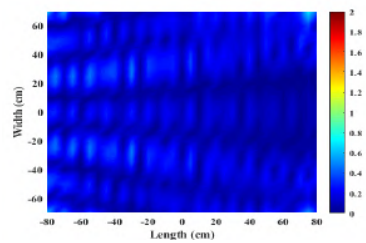

Err(PlanE,Zone,H)

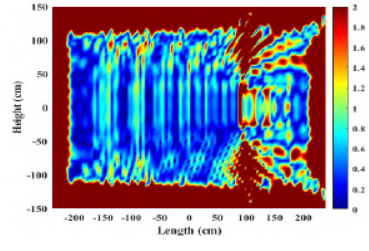

Err(PlanH,Chm,H)

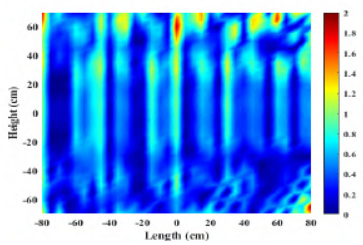

Err(PlanH,Zone,H)

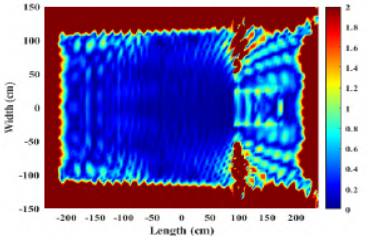

Err(PlanE,Chm,V)

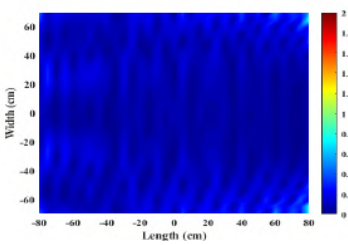

Err(PlanE,Zone,V)

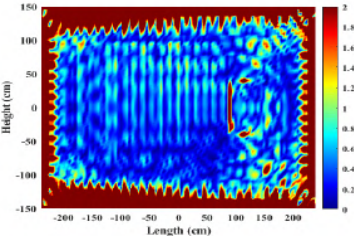

Err(PlanH,Chm,V)

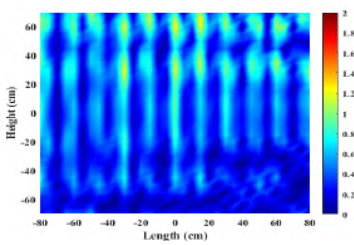

Err(PlanH,Zone,V)

Figure 6. Error in the full length of chamber and in the quiet zone at $1 \mathrm{GHz}$ for a dipole source 


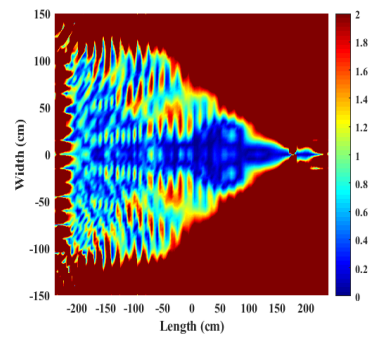

AR(PlanE,Chm,Dipole)

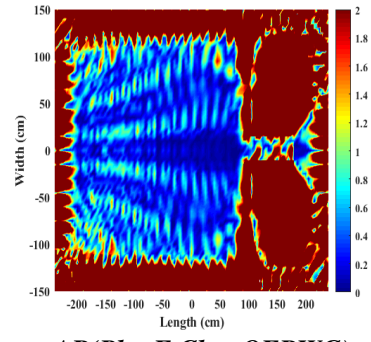

AR(PlanE,Chm,OERWG)

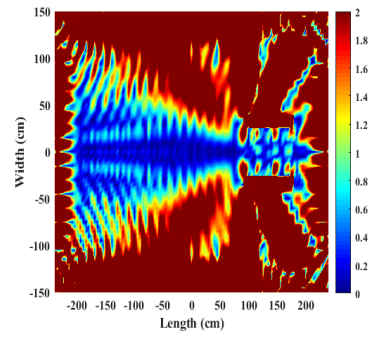

AR(PlanE,Chm,Horn)

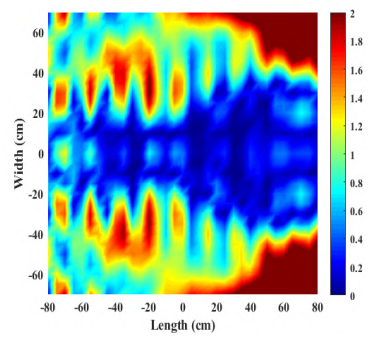

AR(PlanE,Zone,Dipole)

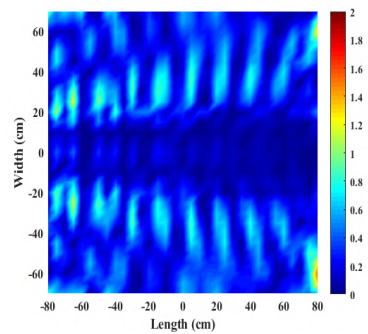

AR(PlanE,Zone,OERWG)

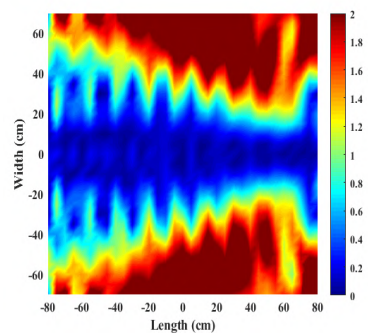

AR(PlanE,Zone,Horn)

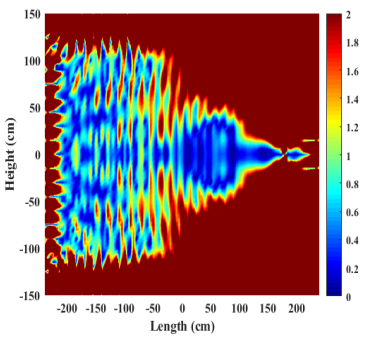

AR(PlanH,Chm,Dipole)

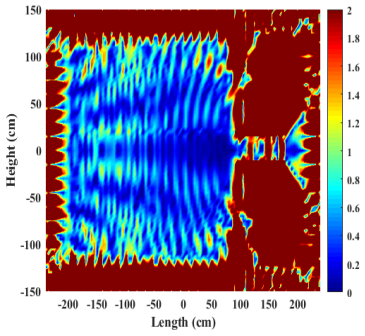

AR(PlanH,Chm,OERWG)

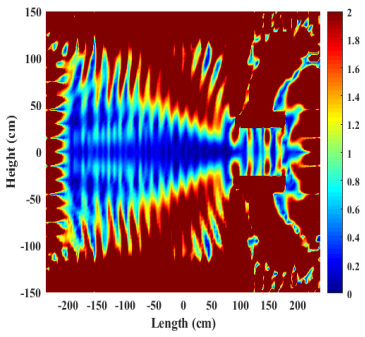

AR(PlanH,Chm,Horn)

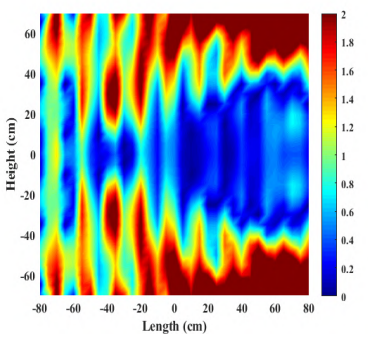

AR(PlanH,Zone,Dipole)

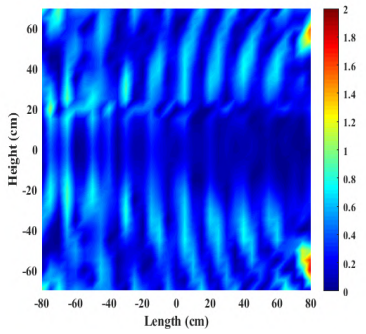

AR(PlanH,Zone, OERWG)

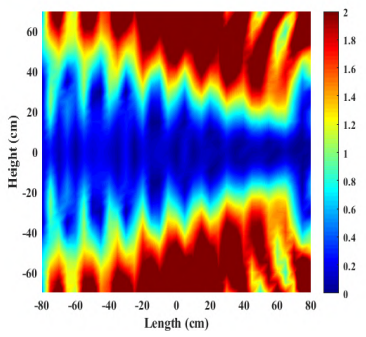

AR(PlanH,Zone,Horn)

Figure 7. Axial ratio in the full length of chamber and in the quiet zone at $1 \mathrm{GHz}$ for a dipole, OERWG and Horn source

\section{CONCLUSION}

In this paper, we have presented a novel methodology for the modeling and characterization of a full anechoic chamber for antenna measurements. This approach is based on the time domain resolution of Maxwell's equations with the method of finite integrals technique. The reduction in computing time and IT resources was achieved by replacing the traditional process of excitation of the antennas by direct excitation of the structure of the chamber by the equivalent Huygens sources of these antennas. This novel technique allowed us to reduce the total simulation time and also the need for IT resources by $90 \%$.

The stability and convergence of our approach were ensured by an autoregressive filter. The results obtained show that the chamber error calculated by the ratio between the field in free space and the field in the chamber as well as the axial ratio given by the ratio of vertical components to horizontal components are comparable with those published in the literature and catalogs of anechoic chamber manufacturers.

In order to prove the validity of our approach, the behavior of the chamber will be studied, in the low frequencies where the absorbers are of large size and in the high frequency where the structure requires a very fine mesh. In addition, the chamber performances will be investigated as function of the geometric form of the absorbers. Others anechoic chambers forms, as semi-anechoic and compact chambers are under test to proven our novel approach.

\section{REFERENCES}

[1] Taybi, C., Moutaouekkil, M. A., Rodrigues, K. K., Elmagroud, B., and Ziyyat, A, "Probes correction for planar near field antennas measurements," in 2015 IEEE 15th Mediterranean Microwave Symposium (MMS), jan 2015, pp. 1-4. 
[2] Kwate, R. K., Derkaoui, A., Elmagroud, B., Taybi, C., Ziyyat, A., and Picard, D, "Non-invasive estimation of whole body averaged SAR by the personal RF dosimeter measurements," in 2019 7th Mediterranean Congress of Telecommunications (CMT), Fès, Morocco, 2019, pp. 1-4.

[3] Meng, D., Li, X., and Gao, X, "Near-field corrections to antenna factor of dipole antennas calibrated in very narrow OATS," in International Conference on Microwave and Millimeter Wave Technology, ICMMT 2010, Chengdu, China, 2010, pp. 1869-1872.

[4] L. H. Hemming, "Electromagnetic Anechoic Chambers: A Fundamental Design and Specification Guide," Wiley Interscience, 2002

[5] C. A. Balanis, "Antenna Theory: Analysis and Design," 3rd ed. Wiley, 2012.

[6] S. Celozzi, R. Araneo, and G. Lovat, "Electromagnetic Shielding. Wiley," 2008.

[7] V. Rodriguez, "Validation of a Method for Predicting Anechoic Chamber Performance: A Technique That Uses Polynomial Approximations for RF Absorber Reflectivity," IEEE Antennas and Propagation Magazine, vol. 60, no. 4, pp. 31-40, aug 2018.

[8] Taybi, C., Derkaoui, A., Khalid, S., Moutaouekkil, M. A., Elmagroud, B., and Ziyyat, A, "Geometrical and Analytical Methods for Probes Correction applied to Near Field Antennas Measurement Systems," in 7th Mediterranean Congress of Telecommunications 2019, CMT 2019, Fès, Morocco, 2019, pp. 1-5.

[9] M. Gillette, "RF anechoic chamber design using ray tracing," in Antennas and Propagation Society International Symposium, Stanford, CA, USA, jun 1977, pp. 246-249.

[10] M. K. Mansour and J. Jarem, "Anechoic chamber design using ray tracing and theory of images," in Conference Proceedings-IEEE SOUTHEASTCON, vol. 2, New Orleans, LA, USA, 1990, pp. 689-695.

[11] D. Campbell, G. Gampala, M. Vogel, and C. Reddy, "Simulating antenna measurements in an anechoic chamber," Microwave Journal, vol. 57, no. 7, pp. 90-100, 2014.

[12] D. Campbell and Gampal, "Modeling and analysis of anechoic chamber using CEM tools," Applied Computational Electromagnetics Society Journal, vol. 28, no. 9, pp. 755-762, 2013.

[13] Kawabata, M., Ishida, Y., Shimada, K., and Kuwabara, N, "FDTD Method for Site Attenuation Analysis of Compact Anechoic Chamber Using Large- Cell Concept," IEEE Transactions on Fundamentals and Materials, vol. 125, no. 10, pp. 784-790, 2005.

[14] Gradoni, G., Moglie, F., Pastore, A. P., and Primiani, V. M, "Numerical and experimental analysis of the field to enclosure coupling in reverberation chamber and comparison with anechoic chamber," IEEE Transactions on Electromagnetic Compatibility, vol. 48, pp. 203-211, 2006.

[15] G. Dun, "Modélisation et optimisation de chambres anécho ıques pour applications CEM," Ph.D. disser- tation, Bretagne Occidentale, 2007.

[16] F. Bellamine, "Analysis of semi-anechoic chambers using the frequency dependent transmission line modeling," in European Space Agency, vol. 626 SP, 2006.

[17] B. F, "Simulation of anechoic chambers using a combined TLM and homogenisation method in the frequency range 30-200 MHz," in First European Conference on Antennas and Propagation, nov 2008, pp. 1-6.

[18] R. Maleewan and S. Thitirat, "Deep Learning Based Gesture Classification for Hand Physical Therapy Interactive Program," Lecture Notes in Computer Science, vol. 12198, pp. 349-358, 2020.

[19] X. Tianqi, "A Computer Vision Based Beamforming Scheme for Millimeter Wave Communication in LOS Scenarios," in 7th International Conference on Computer Science and Network Technology, Oct 2019, pp. 1-6.

[20] Xie, L., Wang, C., Bu, Y., Sun, J., Cai, Q., Wu, J., and Lu, S, “An RFID-Based Approach for Recognition of Multiple Tagged Objects in Augmented Reality Systems," IEEE Transactions on Mobile Computing, vol. 15, pp. 1188-1202, 2020.

[21] Taybi, C., Ziyyat, A., Moutaouekkil, M. A., and Elmagroud, B, "Novel Approach for Time-Domain Modeling a Full Anechoic Chamber for NearIFar-Field Antennas Measurement," in International Symposium on Advanced Electrical and Communication Technologies, ISAECT 2018, Rabat, Morocco, 2019, pp. 1-6.

[22] M. Clemens and T. Weiland, "Discrete electromagnetism with the finite integration technique," Progress in Electromagnetics Research, vol. 32, pp. 65-87, 2001.

[23] I. Munteanu and T. Weiland, "RF Microwave Simulation with the Finite Integration Technique-From Component to System Design," in Scientific Computing in Electrical Engineering. Springer, Berlin, Heidelberg, 2007, pp. 247-260.

[24] T. Wittig, R. Schuhmann, and T. Weiland, "Model order reduction for large systems in computational electromagnetics," Linear Algebra and Its Applications, vol. 415, no. 2-3, pp. 499-530, 2006.

[25] M. Mocker, S. Hipp, F. Spinnler, H. Tazi, and T. Eibert, "Comparison of electromagnetic solvers for antennas mounted on vehicles," Advances in Radio Science, vol. 13, pp. 49-55, 2015.

[26] A. Smirnov, "Procedural aspects of the certification of anechoic chambers," Measurement Techniques, vol. 53, no. 5, pp. 556-562, 2010.

[27] TDK, "Fully Compliant Anechoic Chambe," Tech. Rep., 2016.

[28] ETS-Lindgren, "ETS-Lindgren Anechoic Chambers," Tech. Rep., 2016. 